\title{
Increased Asymmetric Dimethylarginine and Ischemia-Modified Albumin Levels in Obstructive Sleep Apnea
}

\author{
Aysel Sunnetcioglu MD, Selvi Asker MD, Hamit Hakan Alp MD, and Hulya Gunbatar MD
}

\begin{abstract}
BACKGROUND: Asymmetric dimethylarginine and ischemia-modified albumin are new biomarkers that are used for evaluation of ischemia and oxidative stress. The present study aimed to investigate whether serum levels of asymmetric dimethylarginine and ischemia-modified albumin are altered in subjects with obstructive sleep apnea (OSA). METHODS: A cross-sectional, clinical study was implemented on data derived from 79 subjects who underwent polysomnography. Cases were allocated into 3 groups with respect to polysomnography results: Group 1 consisted of 22 subjects without apnea, whereas Group 2 comprised 29 subjects with mild to moderate OSA, and Group 3 included 28 subjects with severe OSA. These 3 groups were compared in terms of demographic datas and polysomnographic parameters, serum levels of asymmetric dimethylarginine and ischemia-modified albumin. RESULTS: Serum levels of ischemia-modified albumin were significantly higher in Groups 2 and $3(P=.001)$. Mean $\mathrm{S}_{\mathrm{pO}_{2}}$ of Group 3 was notably lower than that of Groups 1 and $2(P<.001)$, whereas times for $\mathrm{S}_{\mathrm{pO}}<90 \%$ were statistically significantly different from each other in all 3 groups $(P<.001)$. Serum levels of asymmetric dimethylarginine in Group 3 were notably higher than those in Group $1(P=.027)$. Levels of ischemiamodified albumin were correlated positively with $\mathrm{AHI}$ and time $\mathrm{S}_{\mathrm{pO}_{2}}<90 \%$ values $(P=.008$ and $P<.001$, respectively). CONCLUSIONS: Ischemia-modified albumin and asymmetric dimethylarginine were significantly higher in subjects with OSA. Furthermore, ischemia-modified albumin was independently associated with severity of OSA defined by AHI and severity of oxygen desaturation. Key words: obstructive sleep apnea; asymmetric dimethylarginine; ischemia modified albumin. [Respir Care 2016;61(8):1038-1043. () 2016 Daedalus Enterprises]
\end{abstract}

\section{Introduction}

Obstructive sleep apnea (OSA) is a common disorder characterized by recurrent episodes of upper airway obstruction during sleep. ${ }^{1}$ Due to intermittent hypoxia, chronic sympathetic activation, and systemic inflammation seen in conjunction with OSA, the risk of cardiovascular morbidity was found to be increased. ${ }^{2-8}$ How-

Drs Sunnetcioglu, Asker, and Gunbatar are affiliated with the Department of Chest Diseases, and Dr Alp is affiliated with the Department of Biochemistry, Medical Faculty, Yuzuncu Yil University, Van, Turkey.

The authors have disclosed no conflicts of interest.

Correspondence: Aysel Sunnetcioglu MD, Department of Chest Diseases, Yuzuncu Yil University, Faculty of Medicine, Van, Turkey. E-mail: izciaysel@mynet.com.

DOI: $10.4187 /$ respcare.04472 ever, the underlying pathophysiology for the link between OSA and cardiovascular disorders has not been precisely elucidated. ${ }^{4,6,7}$

Asymmetric dimethylarginine is the endogenous inhibitor of nitric-oxide synthase. Since asymmetric dimethylarginine inhibits nitric oxide synthase activity, decreased nitric oxide levels result in impaired endothelial function and decreased vasodilatation. Asymmetric dimethylarginine is now known to be a mediator molecule of the adverse vascular effects of many other factors and markers of cardiovascular risk. Asymmetric dimethylarginine levels increase under hypoxic conditions. ${ }^{9}$ Increased asymmetric dimethylarginine concentration indicates endothelial dysfunction and is an important parameter in determining cardiovascular morbidity and mortality.

Ischemia-modified albumin has been regarded as a new and emerging marker of ischemia and oxidative stress. ${ }^{2}$ During ischemia/reperfusion, the structure of the amino terminus of albumin is changed in a way that causes the 
decrease of its cobalt binding capacity, leading to the formation of an ischemia-modified albumin. ${ }^{10}$ It increases within minutes after the onset of ischemia, and its levels remain elevated for several hours and return to normal within 24 h. ${ }^{11}$

Intermittent hypoxia, hypoxia/reoxygenation sequences, and high oxidative stress in patients with OSA are the major events causing endothelial dysfunction. Vascular tone and endothelial function are 2 important factors that may be involved in the association of OSA with cardiovascular morbidity. Impairment of endothelium-dependent vasodilatation had been reflected as changes in levels of nitric oxide and asymmetric dimethylarginine in OSA. ${ }^{8,12}$ Similarly, one study ${ }^{13}$ implied that serum ischemia-modified albumin, an indicator of oxidative stress and inflammation, may serve as a biomarker in OSA. Our hypothesis was that these levels would be elevated. The aim of the present study was to investigate the alteration of serum levels of asymmetric dimethylarginine and ischemia-modified albumin in OSA and to test their correlation with demographic and polysomnographic indices.

\section{Methods}

\section{Study Design}

This cross-sectional, clinical study was conducted in the Pulmonology Department at the Faculty of Medicine at Yuzuncu Yil University Hospital (Van, Turkey) between January and June 2014. The study was approved by the institutional review board. Seventy-nine subjects with a complaint of excessive daytime sleepiness and snoring were recruited for polysomnography. OSA was defined as apnea-hypopnea index $(\mathrm{AHI}) \geq 5$ events/h. Cases with AHI $<$ 5 constituted Group $1(n=22)$, whereas cases with AHI values between 5 and 30 were termed as mild to moderate OSA (Group 2, $n=29$ ), and subjects with an AHI of $\geq 30$ (severe OSA) constituted Group $3(n=28)$.

Previous treatment for sleep disorder (oral appliances or positive airway pressure before the sleep study), history of uvulopharyngeal or orthognathic surgery, any neurological deficit or neuromuscular diseases, and diagnosis of another sleep disorder, including central apnea, were exclusion criteria for the present study. In addition, patients with hypertension, diabetes mellitus, hyperlipidemia, COPD, interstitial lung disease, or asthma were also excluded from the study.

\section{Sleep Study}

Diagnosis of OSA was accomplished with respect to the results of overnight polysomnography by means of a digital 16-channel Embla (Medcare, Reykjavik, Ice-

\section{QUICK LOOK}

\section{Current knowledge}

Intermittent hypoxia, hypoxia/reoxygenation sequences, and high oxidative stress in patients with obstructive sleep apnea (OSA) are the major events causing endothelial dysfunction. Asymmetric dimethylarginine and ischemia-modified albumin are produced in response to ischemic stresses, such as hypoxia. However, asymmetric dimethylarginine and ischemia-modified albumin in OSA causing endothelial dysfunction has not been precisely evaluated.

\section{What this paper contributes to our knowledge}

Ischemia-modified albumin and asymmetric dimethylarginine were significantly higher in subjects with OSA. Furthermore, ischemia-modified albumin levels were associated with severity of OSA defined by apnea-hypopnea index. Serum levels of ischemia-modified albumin and asymmetric dimethylarginine may be altered in OSA, reflecting the deterioration of vascular endothelial function.

land) polygraph performed at our sleep laboratory. Generally accepted definitions and scoring methods were utilized, and diagnosis of OSA was established in the presence of chest and abdominal paradoxical movement during apnea events or flow limitation on the nasal pressure signals. ${ }^{1}$

\section{Biochemical Assays}

Blood samples were obtained early in the morning after an overnight fasting period. Peripheral venipuncture was made from brachial veins, and blood samples were collected into vacutainer tubes. Serum samples were centrifuged at $3,000 \mathrm{rpm}$ for $5 \mathrm{~min}$ and were maintained at $-80^{\circ} \mathrm{C}$ until analysis.

Reduced cobalt-to-albumin binding capacity (ischemiamodified albumin level) was measured using the rapid and colorimetric method developed by Bar-Or et al ${ }^{14}$ Briefly, $200 \mu \mathrm{L}$ of subject serum was transferred into glass tubes, and $50 \mu \mathrm{L}$ of $0.1 \% \mathrm{CoCl}_{2} \times 6 \mathrm{H}_{2} \mathrm{O}$ (Sigma-Aldrich, St. Louis, Missouri) was added. After gentle shaking, the mixture was incubated for $10 \mathrm{~min}$ to ensure sufficient cobaltalbumin binding. Then $50 \mu \mathrm{L}$ of $1.5 \mathrm{mg} / \mathrm{mL}$ dithiothreitol (Sigma-Aldrich) was added as a coloring agent. After $2 \mathrm{~min}$, $1 \mathrm{~mL}$ of $0.9 \% \mathrm{NaCl}$ was added to halt the binding between the cobalt and albumin. A blank was prepared for every specimen. At the dithiothreitol addition step, $50 \mu \mathrm{L}$ of distilled water was used instead of $50 \mu \mathrm{L}$ of $1.5 \mathrm{mg} / \mathrm{mL}$ 


\section{Asymmetric Dimethylarginine and Ischemia-Modified Albumin in OSA}

Table 1. Comparison of Demographic, Polysomnographic, and Biochemical Variables Under Investigation in the 3 Subject Groups

\begin{tabular}{|c|c|c|c|c|}
\hline Variable & Group $1(n=22)$ & Group $2(n=29)$ & Group $3(n=28)$ & $P$ \\
\hline Male/female sex, $n$ & $14 / 8$ & $22 / 7$ & $21 / 7$ & .58 \\
\hline Age, mean \pm SD y & $35.8 \pm 9.5$ & $46.2 \pm 10.0$ & $47.0 \pm 14.0$ & $<.001$ \\
\hline $\mathrm{BMI}$, mean $\pm \mathrm{SD} \mathrm{kg} / \mathrm{m}^{2}$ & $27.8 \pm 3.9$ & $31.3 \pm 5.7$ & $33.1 \pm 7.0$ & .008 \\
\hline $\mathrm{AHI}$, mean $\pm \mathrm{SD} \mathrm{h}$ & $2.2 \pm 1.1$ & $15.6 \pm 6.2$ & $71.3 \pm 28.2$ & $<.001$ \\
\hline $\mathrm{REM} \mathrm{AHI}$, mean $\pm \mathrm{SD} \mathrm{h}$ & $2.5 \pm 2.4$ & $2.9 \pm 2.3$ & $4.7 \pm 2.5$ & .005 \\
\hline Non-REM AHI, mean $\pm \mathrm{SD}$ h & $2.0 \pm 1.2$ & $15.0 \pm 9.1$ & $72.4 \pm 31.0$ & .001 \\
\hline $\mathrm{ODI}$, mean $\pm \mathrm{SD}$ & $2.5 \pm 1.7$ & $15.3 \pm 8.0$ & $60.3 \pm 27.9$ & .001 \\
\hline Number of apneas, mean $\pm \mathrm{SD}$ & $2.7 \pm 2.5$ & $20.5 \pm 15.3$ & $275.0 \pm 242.4$ & $<.001$ \\
\hline Times for $\mathrm{S}_{\mathrm{pO}_{2}}<90 \% / \mathrm{min}$, mean $\pm \mathrm{SD} \%$ & $0.15 \pm 2.55$ & $11.40 \pm 34.30$ & $46.35 \pm 41.30$ & $<.001$ \\
\hline Mean $\mathrm{S}_{\mathrm{pO}_{2}}$, mean $\pm \mathrm{SD} \%$ & $92.60 \pm 3.05$ & $91.60 \pm 2.10$ & $87.10 \pm 15.28$ & $<.001$ \\
\hline IMA, mean \pm SD units $/ \mathrm{L}$ & $0.84 \pm 0.49$ & $1.98 \pm 1.43$ & $2.28 \pm 1.00$ & .001 \\
\hline $\mathrm{ADMA}$, mean $\pm \mathrm{SD} \mu \mathrm{mol} / \mathrm{L}$ & $1.54 \pm 0.35$ & $1.69 \pm 0.15$ & $1.72 \pm 0.20$ & .027 \\
\hline $\begin{array}{l}\mathrm{BMI}=\text { body mass index } \\
\mathrm{AHI}=\text { apnea-hypopnea index } \\
\mathrm{REM}=\text { rapid eye movement } \\
\mathrm{ODI}=\text { oxygen desaturation index } \\
\text { Times for } \mathrm{S}_{\mathrm{pO}_{2}}<90 \%=\text { time arterial oxygen saturation } \\
\mathrm{IMA}=\text { ischemia-modified albumin } \\
\mathrm{ADMA}=\text { asymmetrical dimethylarginine }\end{array}$ & & & & \\
\hline
\end{tabular}

dithiothreitol to obtain a blank without dithiothreitol. The absorbances were recorded at $470 \mathrm{~nm}$ with a UV1601 spectrophotometer (Shimadzu, Kyoto, Japan). Color formation in specimens with dithiothreitol was compared with color formation in the blank tubes, and the results were expressed as absorbance units. Serum levels of asymmetric dimethylarginine were detected with a commercially available enzyme-linked immunosorbent assay kit. Measurement was performed using a computer-based enzymelinked immunosorbent assay reader (ELx 800 Universal Microplate Reader, Biotek Instruments, Winooski, Vermont) with a wavelength of $405 \mathrm{~nm}$.

\section{Outcome Parameters}

Demographic data (age, sex, body mass index), polysomnographic indices (AHI, mean oxygen saturation, time for arterial oxygen saturation $\left[\mathrm{S}_{\mathrm{pO}_{2}}\right]<90 \%$, number of apnea), and biochemical parameters (serum levels of asymmetric dimethylarginine and ischemia-modified albumin) were noted and compared in 3 groups. Moreover, correlations of biochemical parameters to demographic and polysomnographic variables were sought.

\section{Statistical Analysis}

Data were analyzed using SPSS Statistics 20.0 for Windows (IBM, Armonk, New York). Normal distribution of variables was assessed with the Kolmogorov-Smirnov test. Parametric methods were used in the analysis of the variations that have normal distribution, and nonparametric methods were used in the analysis of the variations that do not have normal distribution. Correlation between variables with normal distribution was evaluated with the Pearson correlation test, whereas Spearman's rho test was preferred if at least one of the variables did not exhibit normal distribution. Comparison of groups in terms of variables with normal distribution was implemented with one-way analysis of variance. Variables without normal distribution were evaluated with the Kruskal-Wallis test. Homogeneity of variances of groups was analyzed with the Levene test. For groups with variables with homogeneous variances, the Duncan test was used. However, the Tamhane test was preferred for variables of groups with non-homogeneous variances. Quantitative variables were expressed as mean, SD, minimum, and maximum values. CI was set at $95 \%$, and all differences associated with a $P$ value of $<.05$ were considered statistically significant.

\section{Results}

A comparison of variables under investigation in the 3 groups is shown in Table 1. Distribution of male/female subjects was similar in the 3 groups $(P=.58)$. Those in Group 1 were younger than those in Groups 2 and 3 $(P<.001)$, but there was no age difference between those in Groups 2 and $3(P=.99)$. The body mass index of Group 1 was remarkably lower than those of Groups 2 and $3(P=.008)$. Serum levels of ischemia-modified albumin were significantly higher in Groups 2 and $3(P=.001)$. Levels of serum ischemia-modified albumin in the 3 groups are shown in Figure 1. Mean oxygen saturation of Group 3 was notably lower than that of Groups 1 and $2(P<.001)$, whereas times for $\mathrm{S}_{\mathrm{pO}_{2}}<90 \%$ were statistically signifi- 


\section{Asymmetric Dimethylarginine and Ischemia-Modified Albumin in OSA}

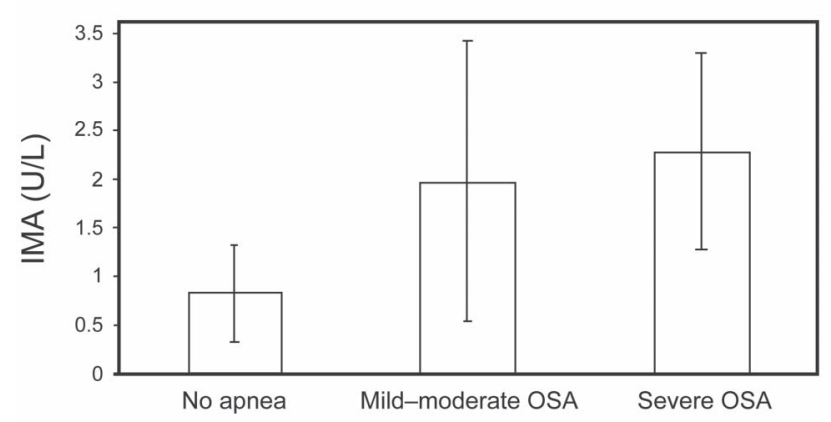

Fig. 1. Serum ischemia-modified albumin (IMA) levels in the 3 subject groups. $P=.001$.

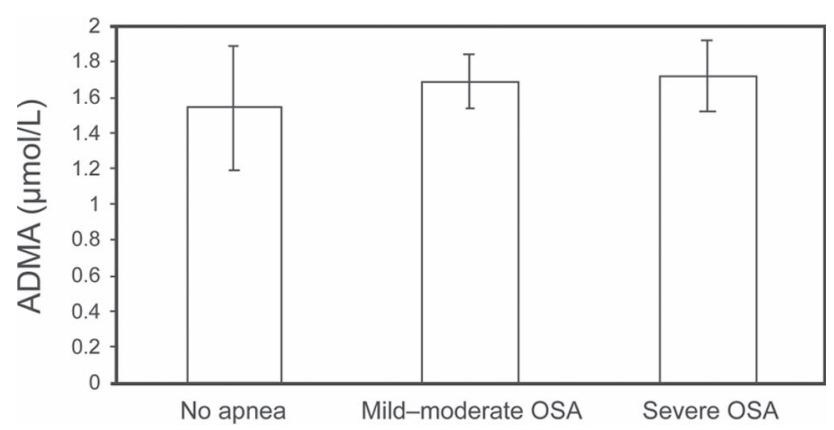

Fig. 2. Serum asymmetric dimethylarginine (ADMA) levels in the 3 subject groups. $P=.027$.

cantly different from each other in all 3 groups $(P<.001)$. Serum levels of asymmetric dimethylarginine in Group 3 were notably higher than that in Group $1(P=.027)$. Levels of serum asymmetric dimethylarginine in the 3 groups are demonstrated in Figure 2. Correlation analysis revealed that only serum levels of ischemia-modified albumin were correlated positively with AHI and time for $\mathrm{S}_{\mathrm{pO}_{2}}<90 \%$ values $(\mathrm{r}=0.298, P=.008$ and $\mathrm{r}=0.458$, $P=.001)$. In subjects with OSA, there was no correlation between ischemia-modified albumin and asymmetric dimethylarginine $(\mathrm{r}=0.75, P=.51)$.

\section{Discussion}

The present study was carried out to investigate whether serum levels of asymmetric dimethylarginine and ischemia-modified albumin were altered in OSA and to determine whether levels of these biomarkers were correlated with demographic parameters or polysomnographic indices. Our results have shown that serum levels of ischemiamodified albumin and asymmetric dimethylarginine were significantly higher in subjects with OSA, and levels of ischemia-modified albumin were correlated positively with AHI and values for time $\mathrm{S}_{\mathrm{pO}_{2}}<90 \%$.

Obstructive sleep apnea is reported to be a risk factor for cardiovascular morbidity due to the hazardous out- comes of oxidative stress, vascular endothelial dysfunction, and inflammation. ${ }^{15-18}$ Efforts made to clarify the link between OSA and cardiovascular disorders have pointed to endothelial dysfunction as a key step linking these 2 pathological processes. ${ }^{16-18}$ Chronic intermittent hypoxia may have reverse effects on endothelial function, reducing endothelial nitric oxide bioavailability and amplifying the effects of oxidative stress and inflammation. ${ }^{19,20}$ Increased levels of adhesion molecules and hypercoagulability enhance the vascular damage, leading to the occurrence of cardiovascular morbidity in OSA.19,20

Asymmetric dimethylarginine levels were increased under hypoxic conditions. ${ }^{9}$ In addition to oxidative stress, elevated asymmetric dimethylarginine levels are substantial factors leading to impairment of nitric oxide availability and diminished endothelium-dependent vasodilatation, and their increased levels have been reported in cardiovascular disease. ${ }^{21,22}$ Increased plasma concentration of asymmetric dimethylarginine is associated with hypertension, pulmonary hypertension, hypercholesterolemia, and severe peripheral artery occlusive diseases. ${ }^{15,17,23,24}$ These findings suggest that asymmetric dimethylarginine is responsible for endothelial dysfunction. Barceló et $\mathrm{al}^{25}$ assessed plasma asymmetric dimethylarginine in subjects with OSA and found it to be significantly higher in these subjects irrespective of the presence of further cardiovascular risk. Following CPAP therapy with significant reduction of intermittent hypoxemia, the levels of asymmetric dimethylarginine can be decreased. ${ }^{15}$

Ischemia-modified albumin may indicate underlying subclinical disease or vascular dysfunction. ${ }^{26}$ Increased levels of ischemia-modified albumin may serve as a nonspecific risk marker for evaluation of the oxidative stress status and atherosclerotic burden. ${ }^{13,27,28}$ Increased production of free radicals and pro-inflammatory cytokines in OSA are sources of the oxidative damage at the cellular and subcellular levels. ${ }^{18}$ Yang et $\mathrm{al}^{13}$ suggested that increased levels of ischemia-modified albumin in subjects with OSA may result from increased oxidative stress. Ischemia-modified albumin is produced in response to ischemic stresses, such as hypoxia and acidosis. The attenuation of ischemia-modified albumin frequently indicates an acute ischemic event like acute coronary syndrome, a pulmonary embolism, or acute ischemic stroke. ${ }^{29-31}$ Similarly, our results demonstrated that serum ischemia-modified albumin levels were elevated significantly in all subjects with OSA. Correlation of ischemia-modified albumin levels with oxygen desaturation index and $\mathrm{AHI}$ is interesting, since it suggests that indicators of systemic inflammation may reflect the link between OSA and cardiovascular morbidity. Further trials must be carried out to investigate the predictive potential of ischemia-modified albumin for severities of oxygen desaturation and OSA. 


\section{Asymmetric Dimethylarginine and Ischemia-Modified Albumin in OSA}

A study done by Verma at al. ${ }^{32}$ on the role of ischemiamodified albumin in pathogenesis of hyperthyroidism has indicated that elevated ischemia-modified albumin is associated with increased production of asymmetric dimethylarginine, which acts as an inhibitor of nitric-oxide synthase. This may account for ischemic injury caused by the reduction of nitric oxide synthesis. We could not find a correlation between ischemia-modified albumin and asymmetric dimethylarginine in the subjects with OSA.

\section{Conclusions}

Ischemia-modified albumin and asymmetric dimethylarginine were significantly higher in subjects with OSA. Furthermore, ischemia-modified albumin was associated with severity of OSA syndrome defined by AHI and severity of oxygen desaturation. Results of the current study have shown that serum levels of ischemia-modified albumin and asymmetric dimethylarginine may be altered in OSA, reflecting the deterioration of vascular endothelial function. Understanding the clinical importance of these potential biomarkers and their utility in terms of diagnosis, follow-up, and treatment will necessitate further clinical trials on larger series.

\section{REFERENCES}

1. Berry RB, Brooks R, Gamaldo CE, Harding SM, Lloyd RM, Marcus $\mathrm{CL}$, et al. The AASM manual for the scoring of sleep and associated events: terminology and technical specifications, version 2.0.2. www.aasmnet.org. Darien, IL: American Academy of Sleep Medicine, 2013.

2. Strohl KP, Brown DB, Collop N, George C, Grunstein R, Han F, et al. An official American Thoracic Society Clinical Practice Guideline: sleep apnea, sleepiness and driving risk in noncommercial drivers: an update of a 1994 statement. Am J Respir Crit Care Med 2013;187(11):1259-1266.

3. Pack AI, Gislason T. Obstructive sleep apnea and cardiovascular disease: a perspective and future directions. Prog Cardiovasc Dis 2009;51(5):434-451.

4. Bradley TD, Floras JS. Obstructive sleep apnoea and its cardiovascular consequences. Lancet 2009;373(9657):82-93.

5. Chowdhury M, Adams S, Whellan DJ. Sleep-disordered breathing and heart failure: focus on obstructive sleep apnea and treatment with continuous positive airway pressure. J Card Fail 2010;16(2): 164-174.

6. Bordier P. Sleep apnoea in patients with heart failure: part II: therapy. Arch Cardiovasc Dis 2009;102(10):711-720.

7. Bhadriraju S, Kemp CR Jr, Cheruvu M, Bhdriraju S. Sleep apnea syndrome: implications on cardiovascular diseases. Crit Pathw Cardiol 2008;7(4):248-253.

8. Carlson JT, Rångemark C, Hedner JA. Attenuated endothelium dependent vascular relaxation in subjects with sleep apnoea. J Hypertens 1996;14(5):577-584

9. Yildirim AO, Bulau P, Zakrzewicz D, Kitowska KE, Weissmann N, Grimminger F, et al. Increased protein arginine methylation in chronic hypoxia: role of protein arginine methyltransferases. Am J Respir Cell Mol Biol 2006;35(4):436-443.
10. Oettl K, Stauber RE. Physiological and pathological changes in the redox state of human serum albumin critically influence its binding properties. Br J Pharmacol 2007;151(5):580-590.

11. Sbarouni E, Georgiadou P, Kremastinos DT, Voudris V. Ischemia modified albumin: is this marker of ischemia ready for prime time use? Hellenic J Cardiol 2008;49(4):260-266.

12. Dean RT, Wilcox I. Possible atherogenic effects of hypoxia during obstructive sleep apnea. Sleep 1993;16(8 Suppl):S15-S21; discussion S21-S22.

13. Yang LX, Ma SG, Liu H, Xu W. Influence of obstructive sleep apnea on serum butyrylcholinesterase activity and ischemia-modified albumin levels. Clinics 2013;68(7):968-973.

14. Bar-Or D, Lau E, Winkler JV. A novel assay for cobalt-albumin binding and its potential as a marker for myocardial ischemia a preliminary report. J Emerg Med 2000;19(4):311-315.

15. Ohike Y, Kozaki K, Iijima K, Eto M, Kojima T, Ohga E, et al. Amelioration of vascular endothelial dysfunction in obstructive sleep apnea syndrome by nasal continuous positive airway pressure-possible involvement of nitric oxide and asymmetric NG, NG-dimethylarginine. Circ J 2005;69(2):221-226.

16. Gozal D, Kheirandish-Gozal L, Serpero LD, Sans Capdevila O, Dayyat E. Obstructive sleep apnea and endothelial function in schoolaged nonobese children: effect of adenotonsillectomy. Circulation 2007;116(20):2307-2314.

17. Barceló A, Piérola J, de la Peña M, Esquinas C, Sanchez-de la Torre M, Ayllón O, et al. Day-night variations in endothelial dysfunction markers and haemostatic factors in sleep apnoea. Eur Respir J 2012; 39(4):913-918.

18. Hoyos CM, Melehan KL, Liu PY, Grunstein RR, Phillips CL. Does obstructive sleep apnea cause endothelial dysfunction? a critical review of the literature. Sleep Med Rev 2015;20:15-26.

19. Lavie L, Hefetz A, Luboshitzky R, Lavie P. Plasma levels of nitric oxide and L-arginine in sleep apnea subjects: effects of nCPAP treatment. J Mol Neurosci 2003;21(1):57-63.

20. Lavie L. Oxidative stress inflammation and endothelial dysfunction in obstructive sleep apnea. Front Biosci (Elite Ed) 2012;4(1):13911403.

21. Ozkan Y, Ozkan E, Simşek B. Plasma total homocysteine and cysteine levels as cardiovascular risk factors in coronary heart disease. Int J Cardiol 2002;82(3):269-277.

22. Valkonen VP, Paiva H, Salonen JT, Lakka TA, Lehtimäki T, Laakso J, Laaksonen R. Risk of acute coronary events and serum concentration of asymmetric dimethylarginine. Lancet 2001;358(9299): 2127-2138.

23. Ozkan Y, Firat H, Simşek B, Torun M, Yardim-Akaydin S. Circulating nitric oxide (NO), asymmetric dimethylarginine (ADMA), homocysteine and oxidative status in obstructive sleep apnea-hypopnea syndrome (OSAHS). Sleep Breath 2008;12(2):149-154.

24. Yüksel M, Okur HK, Pelin Z, Öğünç AV, Öztürk L. Arginase activity and nitric oxide levels in subjects with obstructive sleep apnea syndrome. Clinics 2014;69(4):247-252.

25. Barceló A, de la Peña M, Ayllón O, Vega-Agapito MV, Piérola J, Pérez G, et al. Increased plasma levels of asymmetric dimethylarginine and soluble CD40 ligand in subjects with sleep apnea. Respiration 2009;77(1):85-90.

26. Borderie D, Allanore Y, Meune C, Devaux JY, Ekindjian OG, Kahan A. High ischemia-modified albumin concentration reflects oxidative stress but not myocardial involvement in systemic sclerosis. Clin Chem 2004;50(11):2190-2193.

27. Turedi S, Cinar O, Yavuz I, Mentese A, Gunduz A, Karahan SC, et al. Differences in ischemia-modified albumin levels between end stage renal disease subjects and the normal population. J Nephrol 2010;23(3):335-340. 


\section{Asymmetric Dimethylarginine And Ischemia-Modified Albumin In OSA}

28. Ma SG, Wei CL, Hong B, Yu WN. Ischemia-modified albumin in type 2 diabetic subjects with and without peripheral arterial disease. Clinics 2011;66(10):1677-1680.

29. Pantazopoulos I, Papadimitriou L, Dontas I, Demestiha T, Iakovidou $\mathrm{N}$, Xanthos T. Ischaemia modified albumin in the diagnosis of acute coronary syndromes. Resuscitation 2009;80(3):306-310.

30. Turedi S, Gunduz A, Mentese A, Topbas M, Karahan SC, Yeniocak $\mathrm{S}$, et al. The value of ischemia-modified albumin compared with d-dimer in the diagnosis of pulmonary embolism. Respir Res 2008; 9:49.

31. Abboud H, Labreuche J, Meseguer E, Lavallee PC, Simon O, Olivot $\mathrm{JM}$, et al. Ischemia-modified albumin in acute stroke. Cerebrovasc Dis 2007;23(2):216-220.

32. Verma M, Dahiya K, Ghalaut VS, Soni A, Singh J, Dhupper V. Comparative study of ischemia modified albumin and nitric oxide in hyperthyroidism. J Invest Biochem 2014;3(1):17-20. 